\title{
Cúlíutura
}

Vol. XIV, $N^{\circ} 2$ (2020) pp. 121-140

Recibido: 6 de marzo, 2020

Aceptado: 31 de agosto, 2020

\section{GRUPO DE REFERENCIA ETARIO, RÉGIMEN SIMBÓLICO Y RELIGIOSIDAD. LA RELIGIOSIDAD COMO SISTEMA ABIERTO DE CREENCIAS EN ESTUDIANTES UNIVERSITARIOS*}

Age reference group, symbolic regime and religiosity: religiosity as an open system of beliefs among university students

\author{
Olivia Sánchez García** \\ osanchez@correo.uaa.mx
}

\author{
Fernando Plascencia \\ Martínez**** \\ fplascen@correo.uaa.mx
}

\section{Luis Gerardo Bernal Guzmán ${ }^{* * * * *}$ \\ lbernal@correo.uaa.mx}

Universidad Autónoma de Aguascalientes, México.

\begin{abstract}
Resumen
En este artículo, sus autores pretenden mostrar la relación entre el grupo de referencia etario de estudiantes de licenciatura de la Universidad Autónoma de Aguascalientes y la religiosidad como sistema abierto de creencias opuesto a la religión institucional, expresada como forma del régimen simbólico diurno, en el sentido de Gilbert Durand. Se parte de la teoría de los grupos de referencia, la antropología de la imaginación y la sociología, y la antropología de la religión, para formar un modelo teórico que otorgue sentido a datos recopilados mediante la encuesta cuantitativa muestral, la entrevista cualitativa por muestreo intencional de máxima variedad y autoetnografías, en levantamientos de información realizados en diversos momentos de un proyecto de investigación sobre estudiantes de licenciatura. También se aborda la incidencia de los grupos de referencia en su rendimiento escolar, en el que se contempla la religión como una dimensión relevante del capital
\end{abstract}

\footnotetext{
${ }^{*}$ Artículo asociado al proyecto: PIECU18-6 "Incidencia de los grupos de referencia en la trayectoria académica de estudiantes del Centro de Ciencias Sociales y Humanidades de la UAA" (enero 2018 a diciembre 2020). **

Doctora en Ciencias de la Educación. Jefa del Departamento de Sociología y Antropología, Universidad Autónoma de Aguascalientes (UAA). ORCID: https://orcid.org/0000-0003-3388-4757

*** Doctor en Ciencias Antropológicas, profesor de tiempo completo del Departamento de Sociología y Antropología, UAA. ORCID: https://orcid.org/0000-0002-5664-6260

${ }^{* * * *}$ Licenciado en Historia, UAA. Actualmente es asistente de investigación del proyecto PIECU18-6. ORCID: https://orcid.org/0000-0002-3021-1314
} 
simbólico y la modelación de la conducta. Los resultados muestran la construcción de un régimen simbólico denominado nocturno en la antropología de la imaginación, con raíces grupales etarias de naturaleza abierta, tolerante e inclusiva, el cual es reactivo a toda forma de exclusión, discriminación y represión, que da lugar a diversas formas expresivas, entre ellas la religiosidad según la concibe la sociología de la religión de Ferrarotti (1993).

Palabras clave: régimen simbólico, religiosidad, grupo de referencia, teorías de la religión, estudiantes universitarios.

\begin{abstract}
The authors of this article explore the relationship between the age reference group of undergraduate students at Universidad Autónoma de Aguascalientes and religiosity as an open system of beliefs opposed to institutional religion expressed as a form of the diurnal symbolic regime in the sense provided by Gilbert Durand. The article begins with the theory of reference groups, the anthropology of imagination and sociology, and the anthropology of religion to form a theoretical model that gives meaning to the data compiled through the sample quantitative survey, the qualitative interview by intentional sampling of maximum variety and self-ethnographies, and the information gathering conducted at various points in a research project on undergraduate students. It also addresses the impact of reference groups on their academic performance, looking at religion as a key aspect of symbolic capital and behavior modeling. The results show the construction of a symbolic regime that is called nocturnal in the anthropology of imagination, with age group roots that are open, tolerant and inclusive in nature. Said roots are reactive to all manner of exclusion, discrimination and repression, which gives rise to a range of expressive forms including religiosity as conceived in Ferrarotti's sociology of religion (1993).
\end{abstract}

Keywords: Symbolic regime, religiosity, reference group, theories of religion, university students.

\title{
Introducción
}

Este texto deriva de dos proyectos de investigación sobre la relación entre el capital cultural de los grupos de referencia de estudiantes de licenciatura y su rendimiento académico en la Universidad Autónoma de Aguascalientes (UAA), en México, una institución laica, sin adscripción religiosa oficial. El primero se centró en el grupo familiar de los estudiantes (Sánchez, Plascencia y Tapia, 2017), en una investigación realizada durante los años 2016 y 2017. El segundo produjo un informe anual, de un proyecto vigente, que focalizó a los grupos de referencia extrafamiliares; es decir, ajenos al grupo familiar, con atención especial en los pares, llámense amigos o compañeros de clase de la misma edad (Sánchez, Plascencia y Tapia, 2018).

El objetivo general es mostrar cómo se perfiló el sistema de creencias religiosas de los estudiantes en un sistema simbólico, dejando de lado otros aspectos. Los objetivos particulares son tres: el primero, mostrar la manera en que se fue construyendo el sistema de creencias estudiantil, desde la conceptualización de la religión como Iglesia (Durkheim, 
1968), desarrollada en un campo social de competencia por la oferta de la salvación y la sociodicea que fundamenta las asimetrías sociales (Bourdieu, 2006), hasta la religiosidad como práctica personal, desburocratizada y profunda de lo sagrado, al margen de la hieroburocracia y su regulación (Ferrarotti, 1993). El segundo objetivo compete al grupo de referencia, concepto teórico que se fue imponiendo durante la recolección y el análisis de los datos, proceso en el que el foco de atención se desplazó de la familia al amplio y complejo grupo de la comunidad etaria, con sus dificultades conceptuales. Esto lleva al tercer objetivo particular: describir el régimen simbólico nocturno (Durand, 2004) como cosmovisión abierta, igualitaria, inclusiva y solidaria que acompaña al talante igualitario y tolerante del conjunto generacional de edades semejantes, construido como muestra intencional homogénea con la investigación, por un lado; y a la religiosidad como sistema abierto de creencias, por otro. Con esto se intenta poner una dimensión más profunda y comprensiva a lo estudiado desde la antropología de la imaginación.

Nos permitiremos ir señalando en los siguientes acápites, uno por cada objetivo particular, la perspectiva teórica con sus modelos de base y operativos específicos de abordaje, la relevancia y el aporte respectivo y, obviamente, la metodología.

\section{De la religión a la religiosidad}

El primer proyecto de investigación (Sánchez, Plascencia y Tapia, 2016-2017) partió de las nociones de religión y de grupo (de clase, en realidad) provenientes de Bourdieu (2006), para quien:

la religión contribuye a la imposición (disimulada) de los principios de estructuración de la percepción y del pensamiento del mundo y, en particular, del mundo social, en la medida en que ella impone un sistema de prácticas y de representaciones cuya estructura, objetivamente fundada en un principio de división política, se presenta como estructura natural-sobrenatural del cosmos. (2006, p. 37)

La religión es cuestión de imposición, objetiva-subjetiva, cocreadora del habitus de clase, por lo que los estudiantes solo recibirían de su familia el habitus correspondiente de clase, en tanto que la escuela no haría más que reproducir esta condición, al confirmar a los estudiantes de clase alta en su posición, pues tan solo da estado sancionado (títulos y grados) a un estado incorporado, en su sociobiografía de clase, según su ventajosa posición en el campo social. (Bourdieu, 1997; Bourdieu y Passeron, 2003).

La estructura social objetiva se introyecta y coincide con la disposición social subjetiva, por eso es "natural" y evidente al coincidir. La religión definida por Durkheim y por Bourdieu coincide en esa objetividad coaccionante. Bourdieu añade la dimensión subjetiva aparejada, pero esto no sucedió en la investigación, pues los datos lo desmintieron.

La pastoral del privilegio propia del catolicismo de Aguascalientes no vincula tanto como para producir esa eficacia que presume Bourdieu. Los Caballeros de Colón, el Frente Nacional por la Familia Aguascalientes, grupos católicos conservadores de derecha y pertenecientes a los sectores sociales más favorecidos, son tanto los beneficiarios del poder y del sentido que administra la Iglesia, como los destinatarios de la legalización de su 
posición privilegiada (Zalpa, 2002, 2006), así como la fuente de la posición estructural asimétrica dominante y la respectiva disposición simbólica hegemónica que Bourdieu diagnostica adecuadamente. Pero esto no se extiende a toda la sociedad de Aguascalientes, al menos no a todos los estudiantes de la UAA.

$\mathrm{El}$ argumento respectivo debe ser estructural y se fundamenta en una encuesta con un muestreo cuantitativo transversal, no experimental y estratificado, de 375 estudiantes sobre una población de 14.606, realizada en el año 2016 (Sánchez y Plascencia, 2017). En el estado de Aguascalientes, el 93,2\% de la población se declara católica (INEGI, 2010), por lo que al menos estadísticamente domina el campo religioso. ${ }^{1}$ Además, hay formas de "habitar el catolicismo" (Olmos, 2017) que son alentadas por la jerarquía católica, las cuales han sido estudiados por Patiño $(2013,2014)$. Hay movimientos de grupos católicos laicos, como los organizadores de peregrinaciones, el grupo Cristo Ilusión Juvenil Que Nos Une a Nuestros Padres (CRIJUNUPA). Además del Movimiento Familiar Cristiano y la Asociación Nacional Pro Superación Personal (ANSPAC), dedicado a mujeres en condición de pobreza surgido de Los Legionarios de Cristo.

En lo que respecta a la UAA, un $72 \%$ de los estudiantes es católico, un $18 \%$ no practica religión alguna y el restante $10 \%$ se reparte en diversas confesiones. Ese dominio relativo del catolicismo en los estudiantes es dudoso cuando solo el 12,3\% -de ese $72 \%$ católico- practica regularmente la religión. Además, de este 12,3\% solo el 5\% lo hace "casi siempre". Cabe señalar que la UAA, como institución no tiene oficialmente adscripción confesional alguna.

La constatación de que no existe una vinculación de la Iglesia católica con la feligresía estudiantil estudiada se corrobora con las respuestas a la pregunta: “En qué crees?”. La muestra resultó tan variopinta como distante de la ortodoxia católica. Un $72 \%$ católico no se corresponde con solo el $57 \%$ que cree en el infierno ni con el 56,5\% que cree en la Virgen de Guadalupe o con el 45,6\% que cree en los milagros. El porcentaje del $72 \%$ no corresponde tampoco con el 42,4\% que cree en Dios. Por otro lado, los estudiantes manifestaron creer en fantasmas, horóscopos, cartomancia y brujería, en porcentajes menores al 23\%. En pocas palabras, no empata el porcentaje de católicos con los porcentajes encuestados de las creencias de la religión que "profesan", y que repite el obispo en su conferencia de prensa semanal y la mayoría de los curas desde el púlpito. Esta situación nos obligó a realizar un estudio en profundidad. Quizá los alumnos desconocían los fundamentos del catolicismo ortodoxo, aunque eso es difícil en una ciudad en la que el obispo de la diócesis cada lunes en conferencia de prensa, con difusión en periódicos y demás medios de comunicación locales, nos recuerda la ortodoxia y la oposición al aborto, a la propaganda protestante, al matrimonio entre personas del mismo sexo, a la incontinencia sexual, al alcoholismo y el libertinaje juvenil; y a las supersticiones y prácticas contrarias a la religión, como la creencia en fantasmas, brujería, horóscopos y todo lo que no sea una buena y sana práctica de la religión. ${ }^{2}$

\footnotetext{
${ }^{1}$ En la misma fuente se ve que Aguascalientes es el tercer estado con más católicos en México, solo debajo de Zacatecas $(94,4 \%)$ y Guanajuato $(94,3 \%)$. Estos estados son circunvecinos entre sí, y el cuarto lugar lo ocupa Jalisco $(93,1 \%)$. Todos están por encima de la media nacional de catolicismo $(83,9)$.

${ }^{2}$ Vale la pena mostrar la postura típica de la Iglesia católica, reflejada en una cita del día 2 de octubre de 2018. En esta afirma monseñor José María de la Torre: "Algunos dicen que ese desprecio a la vida se ha incrementado desde que se ha implementado el aborto en la Ciudad de México. Como que hay un referente paralelo: si ustedes ven el número de víctimas y desaparecidos cómo se ha ido aumentando, como que crecen los abortos, crecen
} 
En 2017 realizamos una entrevista etnosemántica -cuya heurística consiste en la búsqueda del conocimiento cultural de los actores (Spradley, 1979)-, a la que llamaremos en adelante primera encuesta cualitativa. El resultado describió la religiosidad estudiantil (Sánchez y Plascencia, 2017). Con un muestreo intencional de máxima variación (Miles y Huberman, 1986), se eligieron a estudiantes -según su propia autodenominación- católicos, sin religión, espiritualistas trinitarios, adventistas, budistas, mormones, evangélicos, protestantes y a una informante autodefinida como "pagana".

Resumiremos el documento en el que conceptualizamos la religiosidad de los estudiantes analizados (Sánchez y Plascencia, 2017). Estos definen la religión en términos de Durkheim (1968), al concebirla como institución. Por otro lado, perciben lo sagrado como una experiencia personal, íntima y directa sin mediación de la Iglesia. En su visión muy particular, expresaron en su mayoría que la Iglesia católica es tan represiva de las conductas como cómplice de las injusticias sociales y económicas. No es raro por eso que las prácticas y creencias de muchos estudiantes sobre lo divino no sean iguales a las de la Iglesia católica. Sin dirección institucional, se produce un sistema abierto, personal, circunstancial y oportunista, bajo el cual los estudiantes unen los santos, el horóscopo, la brujería o la ciencia y todo lo que fundamente la experiencia trascendente que colme la angustia ante sus límites físicos, cognitivos y morales, cosas que según Geertz (1987) son fundamento de lo sagrado. Por otro lado, temas que condena la Iglesia católica local: aborto, matrimonio entre personas del mismo sexo y sus símbolos respectivos de castigo a la infracción (pecado), como el infierno o la condenación, son relativizadas o ignoradas comúnmente por nuestros informantes. A esto es lo que llamamos religiosidad, un sistema abierto de creencias, alojado en la subjetividad individual y la experiencia sacra sin mediación institucional (Ferrarotti, 1993). Baste por ahora este resumen; en el tercer objetivo se contempla la figuración simbólica de la religiosidad con más detalle.

La UAA no tiene una confesión religiosa oficial y si bien no es novedoso que coexistan diversos grados de adhesión a las instituciones religiosas y diversas confesiones, no deja de ser problema saber por qué sucede. Pensamos que la concepción de lo sacro, los grados de adhesión o distancia con las instituciones religiosas, deriva de lo que Ferrarotti llama religiosidad.

\section{El grupo de referencia etario: la conformación del nosotros de la simbólica nocturna}

En principio el foco estuvo puesto en la familia de los estudiantes, descartando la conceptualización "grupo de referencia". Simplemente observamos su adscripción de clase según la posición dentro del campo social asimétrico en una estructura de clases (Bourdieu, 2002). El uso de esta perspectiva dio resultados muy pobres para determinar los efectos del capital simbólico familiar en el aprovechamiento escolar, puesto que no encontramos el

más crímenes; disminuyen los abortos, disminuyen los crímenes. Por eso pensamos que una de las estrategias para lograr la paz y la reconciliación en México es defender la vida desde el principio hasta el final" (González, 2 de octubre de 2018). En el mismo escrito citado se recuerda que monseñor José María de la Torre Martín llamó "invertidos" a los homosexuales y comparó a los matrimonios igualitarios como uniones entre seres humanos y perros en 2014. 
habitus de la reproducción de clases que determina el éxito escolar aun antes de ingresar a la escuela. No obstante, nos permitió reconocer la importancia del grupo de pares en los jóvenes universitarios.

En la encuesta cuantitativa se había mostrado una tendencia muy importante, referida a que los estudiantes tenían grupos de referencia constituidos por pares etarios -agentes de la misma edad aproximada-, tales como amigos y asociados por diversos consumos culturales unificadores. Otros investigadores ya han descrito esta tendencia de interactuar con amigos o pares etarios, como característica de la adolescencia y la adultez emergente, cuando la familia es sustituida por grupos de pares (Arnett, 2008). Encontramos que un $48 \%$ de los estudiantes elige a sus amigos para hacer conjuntamente un trabajo, mientras que un $34 \%$ escoge a los estudiantes aplicados. Para estudiar en un examen, prefieren en un $42 \%$ a sus amigos casi tanto como a los aplicados (44\%). Además, para realizar un evento social, un $32 \%$ elige a sus amigos en contra de un $31 \%$ que opta por los "socialitos" o populares; para ir a tomar un café, un $84 \%$, escoge a los amigos mientras que un $76 \%$ recurre a ellos para ir a divertirse a un antro y un $77 \%$ para ir de viaje (Sánchez y Plascencia, 2017).

Juntos a estos datos, que ratifican la emergencia del grupo de amigos como sustituto de la familia, tenemos otros datos adicionales que tienen que ver más con el tema tratado: la familia fue el escenario de la práctica de la religión institucional en la niñez. Esto no surgió de la encuesta cuantitativa, pero asomó en la segunda encuesta cualitativa a una muestra de estudiantes exitosos de entre toda la población de licenciatura de la UAA. Se determinó una ecuación que se volvió una hipótesis comprobada: hay una relación entre la práctica de la religión, la familia, la niñez y la inserción en instituciones que actúan como agentes de la religión.

Pepe, un estudiante exitoso que no adhiere a religión alguna nos dice: "Mi familia no practica mucho la religión (...) cuando era niño íbamos a misa toda la familia... bueno, a las bodas, los bautismos y esas cosas en la familia también asistíamos (...) ya cuando crecimos dejamos de ir a misa todos juntos". Agustín, quien tampoco se declara adherente a religión alguna, pero es creyente en un ser superior, nos informa: "Cuando éramos niños, íbamos (la familia) los domingos juntos a misa (...) después, desde la secundaria y la prepa, dejamos de ir juntos a misa y a veces no vamos todos a bodas y eso de la familia”. El caso es que de 23 estudiantes informantes que hablaron del cultivo de la religión, 14 mencionaron expresamente una práctica religiosa regular en familia que se fue diluyendo hasta llegar a extinguirse en la adolescencia y la adultez emergente. Por el contrario, solo dos informantes, Lula y Aldo, hablaron de una práctica religiosa regular en familia hasta la fecha. Lula es católica practicante, Aldo es evangélico. Ambos casos son más bien excepcionales.

En una investigación realizada por Chami, Scudieri e Imhoff (2018) en una escuela judía, estos observaron que se conservaba la concurrencia familiar, el grupo de pares y la escuela, pero en este caso, la identidad y la confesión religiosa empatan y se complementan, cosa que no sucede en los casos de Aldo y Lula, con un grupo de pares religioso homogéneo, con compañeros de estudio con diversidad en la visión del catolicismo y un contexto educativo laico. Para los jóvenes judíos, el paralelismo del grupo etario, familiar y la escuela refuerza su identidad comunitaria. Hay, en nuestro caso, dificultades para la concurrencia de institución educativa, familia y grupo etario de referencia en la práctica de la religión como tal. Aunque, junto a la práctica de la religión, se da la pertenencia a agrupaciones civiles con tintes religiosos: "Estaba en el coro y en otros grupos de la Iglesia" (Sarahí, sin religión, pero 
creyente de lo sacro y lo trascendente). "En los Boy Scouts nos llevaban a misa" (Alberto, también sin religión, pero creyente de la trascendencia). "Mi mamá nos puso (a mis hermanos y a mí) en la doctrina con mis primos para que hiciéramos la primera comunión" (Lula, católica practicante). Hay coincidencia entre institución, religión y cultivo familiar de la religión en la infancia y la niñez, anterior al desplazamiento de la familia por los grupos de pares, lo cual se comprobó en la tercera encuesta cualitativa.

Desde la sociología de la experiencia religiosa y sus claves, a partir de Ros (2018), usamos la metodología cualitativa en la búsqueda de la creencia como evidencia de lo social y de la racionalidad ordinaria, ubicando la fuente de la creencia religiosa en el testimonio. Un grupo de la licenciatura en historia (generación 2014-2018) de la UAA, en el semestre enero-junio de 2017, fue capacitado en la autoetnografía, una metodología cualitativa que combina la investigación y la escritura desde la experiencia personal para comprender la experiencia cultural. La heurística gira alrededor de la pregunta: ¿cómo la experiencia personal ilustra la experiencia cultural y permite que esa cultura sea comprendida? (Ellis, Adams y Bochner, 2010).

A los alumnos se les solicitó que describieran y analizaran sistemáticamente, por medio de la escritura (grafía), su experiencia personal (auto) para entender la experiencia cultural (etnos). Para esto, se solicitó que expresaran sus creencias sobre la religión y sobre el listado de términos acerca de las creencias religiosas y pararreligiosas paralelas a las de la religión (horóscopos, fantasmas, brujería, etc.) que arrojó la encuesta cuantitativa mencionada texto arriba. Posteriormente, se les pidió que escribieran una narración autoetnográfica sobre la experiencia cultural y personal en torno a la consecución de ese conjunto de creencias personales, en tres fases: niñez, adolescencia y adultez emergente actual.

Los resultados arrojaron dos significativos escenarios: el primero, existe un amplio y tolerante criterio de aceptación en lo que respecta a la diversidad religiosa, sexual y cultural, en general. Además de que se da un sistema abierto de creencias que amalgama elementos de la religión, la New Age, la brujería, la creencia en el tarot, los horóscopos, etc. Pero además, hay una animadversión hacia la religión católica al considerarla como una hieroburocracia, y se da una preferencia por el cultivo personal e íntimo de lo sacro. Pervive, entonces, la religiosidad, lo que resta es observar cómo llegaron a esta. Es ilustrativo el siguiente testimonio de Cristóbal, un católico no practicante:

Para mí, la Iglesia católica es una institución más a manera del hombre; la espiritualidad no está institucionalizada, creo yo. Además, se trata de un Estado que responde a intereses y se organiza con estamentos que te inspiran poca espiritualidad, por lo que para mí, entender que la religión se rige por una institución es una idea absurda. Además, la cuestión religiosa es algo íntimo e importante como para dejarlo en interpretaciones y procesos de otras personas.

Eso precisamente es la religiosidad, lo que parece ser la experiencia sacra de un amplio grupo etario que quizá desborde nuestra población de estudio.

Otro escenario relevante es que las trayectorias de los informantes fueron una comprobación, en general, de la relación de la práctica de la religión institucional con la familia, la infancia y la niñez. Del mismo modo, se observa la forma en que se debilita la práctica de la religión con la aparición de la adolescencia y la adultez emergente. 
Contemporáneamente, la familia deja de ser el grupo de referencia fundamental. Sin embargo, no se puede ignorar que hay dos casos en los que la familia no es del todo desplazada como grupo de referencia principal, tales son las experiencias de Lula y de Aldo. Casualmente, estas dos personas son también los informantes más cercanos a la religión institucional y los más distantes del catolicismo popular sincrético y de las creencias pararreligiosas; sin embargo, su visión religiosa no se distancia mucho del régimen simbólico nocturno, como lo veremos en el último acápite de este artículo. Practican, Lula el catolicismo y Aldo, el protestantismo. Lo hacen regularmente y sus grupos de referencia etarios son asociaciones juveniles religiosas, complementos y refuerzos de su postura. ${ }^{3}$ En general, lo que sucede es el abandono de la práctica familiar de la religión.

Andrea (creyente en un ser superior sin religión) escribe en su autoetnografía: "En los años que estuve en secundaria, los problemas aumentaban, y mis calificaciones y rendimiento bajaban. Yo dejé de ir a misa, pues mi mamá ya tampoco se preocupaba por eso". Cristóbal (católico no practicante heterodoxo) dice algo parecido: "Mi familia materna acostumbraba a reunirse todos los domingos para ir a misa y comer juntos. A mí me tocó eso desde que tengo memoria hasta que era un adolescente y dejé de hacerlo". Daniela (atea precoz) redunda el tema: "Desde que tengo uso de razón, mi familia ha sido practicante religiosa, preocupada por cumplir con los sacramentos de la Iglesia católica cada domingo (...) Durante los primeros años de la secundaria fue que me alejé aún más de las prácticas religiosas". En realidad, Daniela no necesitó llegar a la adultez emergente, ni siquiera a la adolescencia plena para desprenderse de la práctica familiar de la religión. Así lo cuenta: "También en este tiempo, como a los 12 años, ya comenzaba a hacer más públicos algunos comentarios en contra de la institución religiosa y a cuestionar la existencia de Dios, y me regañaba mi cuñada, me llamaba hereje".

En general, la entrada a la secundaria y el paso de la preparatoria a la universidad están marcados por el desprendimiento de la familia y de la práctica religiosa en grupo, así como del abandono de grupos e instituciones religiosas católicas como la Doctrina (institución laica que promueve el catolicismo), los Boy Scouts, los grupos juveniles católicos, el coro parroquial y los grupos que promueven la oración laica (Trecenarios, Quincenarios, etc.), sin olvidar que se deja de lado la formación en colegios particulares, en los que se imparte religión.

El problema que se advierte es el uso de la noción de grupo de referencia, empleado ante la insuficiencia de la noción de clase y sus consecuencias reproductivistas en Bourdieu. Ante la falla de predictibilidad frente a los resultados obtenidos, pues no sucede que los alumnos pertenecientes a sectores más favorecidos sean tan católicos como buenos estudiantes ni que los pertenecientes a sectores precarios sean predominantemente estudiantes poco eficientes, usamos un concepto más descriptivo y más susceptible de enlazar lo social con lo pensado, percibido y practicado por los agentes en los escenarios investigados: el concepto de grupo de referencia.

El concepto de grupo de referencia proviene de la psicología social, pero es estudiado en el campo de la sociología por Robert K. Merton (2002a, 2002b). La teoría de los grupos

\footnotetext{
${ }^{3}$ Lula participó tanto en la segunda encuesta cualitativa, a estudiantes exitosos, como en la escritura de la autoetnografía en la tercera encuesta cualitativa. Aldo solo participó en la segunda encuesta, pero proporcionó información longitudinal, al realizar una breve, pero sustanciosa historia de vida sin proponérselo.
} 
de referencia sistematiza los determinantes y las consecuencias de la estimación y de la autoevaluación que los agentes hacen de valores y normas de otros individuos como parámetros de referencia. También es capital la función de conformidad, la manera en que se integra el agente al grupo por medio de la progresiva aceptación que refuerza esa conformidad colectiva. Así se explica la forma en que se afilia e integra un individuo a un grupo, desde sus aspiraciones y quizá en contra del contexto y del grupo de pertenencia que lo comprende. La ortodoxia familiar católica del grupo de pertenencia parece ser descartada con su simbolismo diurno (Durand, 2004), excluyente, hipercodificado y represivo, por el simbolismo nocturno abierto, incluyente, hipocodificado y tolerante, del grupo de pares referencial por parte de los jóvenes estudiados.

La teoría del grupo de referencia comprende la subjetividad y al agente, en tanto que por su alcance intermedio es capaz tanto de atender a lo micro como incorporarse a una dimensión macro. Abreu (2012) presenta el grupo de referencia como una estructura que comprende, en primer lugar, a un conjunto de agentes que se interrelacionan al interior de determinados contextos socioculturales, como la experiencia y la práctica de lo sacro. En segundo lugar, ese conjunto presenta cierta identidad grupal, dada por normas compartidas y categorías sociales determinadas, como tolerancia, apertura e inclusión. En tercer lugar, los valores se expresan como normas, cánones, patrones, hábitos o rutinas que permiten orientar los comportamientos a adoptar, tanto como las preferencias y las formas que evalúan lo anterior. Así se explica la ausencia de la práctica institucional de la misa y el cultivo ecléctico de diversas formas de lo sobrenatural por su valoración, así como la preferencia por el culto personal. En cuarto lugar, el grupo de referencia responde a contextos estructurales o coyunturales como escenarios de la acción. En quinto lugar, la evaluación se da desde el grupo hacia el grupo, según se aspire a pertenecer a un grupo, se rechace o se desee permanecer en uno. Los estudiantes no dejan de pertenecer al grupo de pertenencia familiar, pero su evaluación y aspiración se ha desplazado hacia el grupo de pares, dejando de lado el régimen diurno del simbolismo de la religión institucional. Es conveniente hacer algunas precisiones sobre el grupo de referencia y sus manifestaciones contiguas.

Hay, además, junto al grupo de referencia, el grupo de pertenencia en el que los actores se incluyen espontáneamente por alguna característica innata, como el ser miembro de una familia. Pueden coincidir el grupo de pertenencia y el de referencia si se aceptan y practican, en diverso grado de conformidad, sus normas y valores. Desde la evaluación del agente, hay grupos de referencia en los que se acepta o aspira a la pertenencia, pero hay otros a los que no se desea pertenecer. La evaluación da lugar al grupo de referencia al que se aspira pertenecer, denominado positivo; y al grupo de referencia del que se rechaza la membresía, denominado negativo. En este sentido, la encuesta cuantitativa se realizó durante el periodo comprendido entre octubre y noviembre de 2019, y se aplicó a las 12 licenciaturas del Centro de Ciencias Sociales y Humanidades de la UAA. Esta forma parte de un proyecto de investigación en proceso, liderado por Sánchez, Plascencia y Tapia (2018-2020). Por ahora solo se ha aplicado a una parte de las carreras y solo hay resultados parciales, pero parece que la muestra de datos y su sentido ya se saturó, como sucedió con la difusión de la religiosidad, aunque no la de unidades de población como para dar porcentajes confiables.

Los resultados obtenidos hasta ahora muestran que entre los grupos de referencia negativos con más alto porcentaje se encuentran los siguientes: como puntero el "prianismo" (categoría emic que une a los partidos políticos PRI y PAN en una amplia constelación de 
símbolos de la corrupción política y moral), seguido por algunos grupos católicos de derecha: Frente por la Familia, Caballeros de Colón o Pastoral de Adolescentes.

Un problema que se presentó es si el grupo etario en cuestión es referencial o no, pues quizá sea menos consistente la conformidad y la aspiración con la membresía. Existe una diferenciación entre los pares -el grupo con el que se comparte determinada característica o estatus- y los amigos - grupo de pares con una relación mutuamente valorada-, lo cual trae consecuencias relevantes, según Arnett (2008). Para este autor, los pares, grupo etario correspondiente, tienen poca influencia y da bajo apoyo a los agentes. En cambio, los grupos de amigos tienen tanto una fuerte influencia como un fuerte apoyo a sus integrantes. Por un lado, estimulan las conductas que constituyen la asociación selectiva al mismo tiempo que inhiben las conductas que atentan contra la asociación selectiva. Por otro lado, proporcionan cuatro tipos de apoyo personal que incluyen al miembro en el grupo: el apoyo informativo, consistente en el consejo y la orientación en la solución de problemas personales, desde los valores y visión del grupo; el apoyo instrumental, expresado en la ayuda en los diversos tipos de tareas o actividades prácticas, con medios o instrucciones; el apoyo solidario o compañerismo, figurado como acompañamiento en actividades sociales rutinarias, rituales o lúdicas. Finalmente, proporciona el estímulo que preserva tanto la estima como la autoestima, al felicitar el éxito y reconfortar en el fracaso. Los apoyos se deberán estudiar en un futuro, pero podemos esbozar algunas ideas con datos del proyecto y encuestas sobre lo que aquí nos ocupa.

En sentido estricto, el grupo de referencia sería el de amigos, pues el grupo etario sería más bien un agregado social; sin embargo, el grupo de referencia no tiene que identificarse con el grupo de amigos, el cual no sería más que un tipo de los grupos de referencia, ya que hay una colectividad etaria que no es tan solo un "agregado de situaciones sociales cuyos ocupantes no están en interacción social. Tienen características sociales idénticas -de sexo, edad, situación matrimonial, ingreso, etcétera, pero no están orientadas necesariamente hacia un cuerpo de normas distintivo y común" (Merton, 2002b, p. 366). Por el contrario, hay un conjunto de normas y elaboraciones simbólicas comunes que permite también unas prácticas si no concertadas, sí semejantes y ampliamente compartidas.

La clase social presenta atributos comunes, pero no es un grupo de referencia si no se comparte identidad ni valores ni fines semejantes, aunque sea en diverso grado de conformidad. El grupo etario de pares que estudiamos no solo comparte la edad aproximada, sino también una forma de ver lo sagrado y un conjunto de valores comunes, como la tolerancia, la apertura, el rechazo a la represión y al control de la conciencia, por lo que no es un mero agregado social, sino un eficiente y eficaz grupo de referencia positivo con un claro, entre otros, grupo de referencia negativo común al que no aspiran ni desean pertenecer, en diversos grados, en la representación social que los aglutina: la Iglesia católica como hieroburocracia.

Por otro lado, es posible pertenecer a más de un grupo de referencia. Para esto, se precisa que el grupo sea abierto; es decir, que no condicione la membresía desde la exclusividad en su propio ámbito, como por ejemplo el religioso, o que no sea relevante la pertenencia a otros grupos de referencia de otros ámbitos. La pertenencia al "Club de Amigos de la Ópera”, si existiese en Aguascalientes, quizá no daría problemas para ser miembro de la grey católica (esperamos). El caso es que la Iglesia católica en Aguascalientes amonesta a quienes simpatizan con el matrimonio homosexual y el aborto. Repetidamente hace saber 
que no es buena práctica de la religión la afición a los horóscopos o el tarot, que el cultivo de la brujería y el yoga no son prácticas ingenuas, sino de otras "religiones", según las conferencias semanales del obispado. La Iglesia católica es, desde su jerarquía, un grupo de referencia cerrado que identifica y codifica una amplia variedad de grupos de referencia como negativos según su evaluación, propia de un proyecto monopólico ligado al mercado de la salvación.

En cambio, el grupo de referencia etario de estudiantes investigados cultiva un sistema abierto y tolerante: la religiosidad. Quizá esto se deba en parte a que no es una producción mental que dependa de una institucionalización rígida, jerarquizada y excluyente. La religiosidad se da entre un amplio grupo de iguales, cuestión que pudimos constatar; quizá sea por eso que porten una amplia representación social inclusiva y tolerante. Quizá también tenga que ver con esa manera de ideación abierta, tolerante y optimista con la que parece que estos jóvenes ven el mundo (esto último se tratará en el siguiente acápite). El objetivo correspondiente permitirá desarrollar lateralmente estas últimas ideas para investigaciones ulteriores.

Los cambios de percepción, acción y pensamiento de los jóvenes respecto a la religión no es una mera consecuencia del desarrollo del individuo. Esto es argumentado y mostrado al poner el énfasis en la correlación entre la introyección subjetiva del agente y su posición sociocultural, tanto en la noción de habitus y su correlato grupal, la clase, como en la noción de representación social, con su correlato grupal respectivo, el grupo de referencia. Se da un cambio en el pensamiento, acción y percepción, que va de la religión a la religiosidad, al desarrollarse un grupo referencia etario que comprende el desarrollo del individuo, pero en una situación en la que se encadenan la trayectoria biocultural del sujeto con las condiciones socioculturales que lo comprenden, tanto como reciben los efectos de su agencia.

\section{El régimen simbólico nocturno: del habitus a la representación social}

El éxito académico no es el producto de la mera reproducción social fatal y automática. No hay un habitus diferencial entre los estudiantes exitosos ni mucho menos la necesaria adscripción a la clase dominante en el espacio social. Los estudiantes exitosos comparten una representación social, no un habitus, acerca de lo conveniente y deseable que es la trayectoria vital ideal aparejada a la formación académica y el ejercicio profesional o la conveniencia de vivir con una carrera universitaria, cualquiera que sea la ocupación. A esto se añade una ética vital que valora la puntualidad, la responsabilidad, la constancia, el compromiso y la honradez en sus formas transparentes y anteriores a toda actividad, juicio o percepción de la realidad. Esto se heredó de la familia de los estudiantes estudiados, desde la de un padre pepenador (recolector de material reciclable en basureros) que habita en un cartonal al oriente de la ciudad, hasta la de un padre que vive en un fraccionamiento residencial al norte de la ciudad. Le llamamos ethos:

Parece que es determinante un ethos de responsabilidad, constancia y disciplina, así como de logro de una vida mejor por medio del estudio. Todos tienen esto en su entorno familiar como ideas fundamentales y esquemas de percepción de un habitus que se expresa con prácticas 
estratégicas para lograr el dominio de los diversos campos sociales. (Sánchez y Plascencia, 2017, p. 21)

El concepto de ethos tiene un sentido reproductivista: un desagregado de las prácticas como sentido común, derivado de las mismas y sin la reflexión de la ética y asimilable al habitus (Bourdieu, 2002, nota 33). Por esto decidimos usar el concepto de representación social.

Para Gilberto Giménez (1999) hay dos teorías que destacadamente tratan la introyección subjetiva de la cultura: el habitus de Bourdieu y la teoría de las representaciones sociales de Moscovici. No usaremos el habitus, sino la representación social, esbozada desde Durkheim (1968). Una representación social es una construcción sociocognitiva del sentido común, análoga a la doxa de Thompson (1993). Se define operacionalmente con los elementos relativos a los valores y creencias del grupo de referencia, pero añadiendo una fuerte dosis sociocognitiva: "conjunto de informaciones, creencias, opiniones y actitudes a propósito de un objeto determinado" (Abric, 1994, p. 19). Este acento sociocognitivo se reafirma con la siguiente definición: "una forma de conocimiento socialmente elaborado y compartido, que tiene una intencionalidad práctica y contribuye a la construcción de una realidad común a un conjunto social" (Jodelet, 1989, p. 36).

La representación social es la parte subjetiva del grupo de referencia, el cual se manifiesta como el agente grupal portador de la misma. Giménez enuncia cinco supuestos que corroboran lo antes dicho: en primer lugar, no hay realidad objetiva en sí misma, sino interiorizada y apropiada. Hay al menos dos apropiaciones simbólicas de la realidad por parte de al menos dos agrupaciones de referencias diversas: la religión institucional, con visión diurna, es decir: jerarquizada, hipercodificada y excluyente, del bloque católico conservador, por un lado. Por otro, la versión nocturna: igualitaria, hipocodificada e incluyente, del grupo etario estudiado. En segundo lugar, estas visiones son "apropiadas", puesto que los dos grupos de referencia interiorizan la realidad de formas diversas. El grupo institucional lo hace desde la ideología y la práctica reproductiva; el de los estudiantes, desde la utopía con su praxis contestataria. En tercer lugar los grupos reconstruyen la realidad en sistemas sociocognitivos particulares: lo que para el bloque conservador católico es un pecado, como el aborto o el matrimonio entre personas del mismo sexo, para los jóvenes estudiados es el símbolo de la libertad y la renovación. En cuarto lugar, el sistema cognitivo es el sistema grupal de valores epistémicos, éticos y estéticos, construido por la historia y la ideología propias. La formación de las vocaciones simbólicas diurna institucional o nocturna de los estudiantes estudiados, corresponden a sus trayectorias respectivas en el contexto social asimétrico, diverso y complejo. En quinto lugar, esta realidad apropiada y estructurada constituye para el grupo y el individuo la realidad misma. Esto es producto de las anteriores condiciones, en las que la textura de la representación social se modela simbólicamente desde la experiencia colectiva y grupal, tanto en la práctica reproductiva como en la praxis renovadora, en un contexto histórico determinado. Tenemos, por tanto, dos concepciones que se objetivan en dos "mundos" distintos.

Esa lógica sociocognitiva de la representación del grupo de referencia etario se puede interpretar a la luz de la antropología de la imaginación (Durand, 2004), como un sistema simbólico de naturaleza nocturna, en la que el conocimiento directo de los sentidos es complementado y ordenado por el conocimiento indirecto, derivado de la representación más 
allá de la realidad presente. La representación simbólica permite una actitud reflexiva y teórica sobre el mundo, desde el signo que designa una cosa ausente, denotativa y unívocamente en la actividad consciente y pragmática, hasta el símbolo que se da ante la imposibilidad de que el pensamiento directo capte significados fuera del proceso simbólico y reflexivo en sí. La parte material del signo designa un referente, una cosa. En el símbolo, su parte material se vincula no con las cosas, sino con el sentido en múltiples vías de significación, lo cual es imposible de descubrir por la percepción. A diferencia del signo, que refleja lo sensible, el símbolo instaura el sentido manifestado con un excedente de significación, por lo cual es un medio de conocimiento y no una mera herramienta como el signo.

Si bien una representación es sociocognitiva, pues hay un contexto social y una subjetividad simbólica relacionados, no sucede que exista un determinismo fatal entre el contexto estructural del grupo de referencia y su representación social, en tanto la producción mental no es un epifenómeno que refleje la situación existencial. No hay determinismo sociológico al estilo de Durkheim o Bourdieu que obligue a la mera adaptación social, lo cual da lugar a la ideología que representa, reproduce y justifica la estructura y posición del grupo de referencia, en una situación en la que la interpelación se produce desde la lógica del sistema de dominación. La determinación simbólica no es perfecta. Esto sucede porque en el ámbito de la representación social, con respecto a los condicionamientos sociales y naturales, no hay tanto una determinación radical del condicionamiento y la causa, sino más bien existe el voluntarismo limitado de la reflexión. El dominio de la representación social puede ser afectado por la determinación, pero es también un reducto de la imaginación que equilibra las determinaciones de lo biológico y lo cultural con la reflexión creativa que permite el simbolismo. ${ }^{4}$

El simbolismo tiene una dimensión psicobiológica reducido por Freud (2005) a las pulsiones innatas propias de un monismo pansexualista libidinal, en el que la simbolización se reduce a un epifenómeno, una pulsión tergiversada por la censura. Por su parte, Durand (2004) postula dos reflejos elementales en los neonatos que dan lugar a dos estructuras sensoriomotrices fundamentales del desarrollo "psicobiosociológico". Estas derivan, a su vez, en tres recorridos simbólicos, dos de ellos básicos y uno derivado. La dominante postural y la dominante nutritiva son las fundamentales; la dominante copulativa deriva de la segunda. La dominante postural se origina en el impulso del neonato por alcanzar la verticalidad y poder observar, distinguir y separar. La dominante nutritiva, el instinto alimentario, motiva los movimientos inconscientes de succión y la orientación en la búsqueda de la teta. De esta última dominante se desarrolla la dominante copulativa, rectora del movimiento cíclico

\footnotetext{
${ }^{4}$ Más allá del condicionamiento reproductivo de la clase social, la representación social común a los estudiantes exitosos estudiados, en la segunda encuesta cualitativa, es un ejemplo de esta elaboración simbólica. En el orden del régimen nocturno, concreta un nivel pedagógico lúdico que no es agónico; no es un lugar de la competencia que arroje resultados asimétricos en la interacción entre vencedor y vencido; no transmite la idea de la competencia que arroja jerarquías, propio del régimen diurno, sino del enfrentar la vida con acciones observables y dignas de ser imitadas (miméticas) desde la familia por ser consideradas como provechosas ("nutritivas"). Es, pues, una representación de la pedagogía de la mímesis, dentro del régimen nocturno en que se mezcla la dominante digestiva (diversas clases sociales) y lo solidario (compromiso, responsabilidad ante la sociedad),

Plascencia, 2017; Sánchez y Plascencia, 2017; Sánchez, Plascencia y Alemán, 2018).
} 
iniciado en la succión y madurado en el ritmo del coito. Estas estructuras sensoriomotrices de acomodación producen respectivamente dos regímenes simbólicos: el diurno y el nocturno. El régimen diurno incluye la dominante postural y el régimen nocturno, tanto la dominante nutritiva como la copulativa. En relación con estos conceptos esbozados, haremos el recorrido antropológico -ampliando, definiendo y aplicando la teoría-desde el paso de la naturaleza biológica hasta la cultura, en sus fases sucesivas: reflejo dominante, esquema, arquetipo, símbolo y sistema (signo).

El régimen diurno es luminoso porque la dominante postural busca la claridad y la postura erguida para distinguir, con el objeto de manipular y excluir, por lo que utiliza la materia luminosa y las técnicas que separan y purifican. El instrumento prototípico es la espada, modelo analógico de todo lo que secciona, separa o purifica. Materialización expresiva de la operación mental polémica que, como arma, vence al caos y la oscuridad, la secciona y excluye. En este sentido, el catolicismo oficial es de naturaleza diurna como régimen simbólico, con un Dios personal pero distante, propio de la pastoral del privilegio que la hieroburocracia católica distancia de la población común, irremediablemente perfecto y alejado de las tribulaciones.

El reflejo biológico da lugar al esquema, imagen y concepto que une el gesto corporal inconsciente del reflejo con las representaciones. La dominante postural tiene como esquema fundamental el distinguir, la disyunción excluyente. La separación se opone a la mezcla, el subir contra la caída. Estos esquemas se transforman fácilmente en esquemas verbales: puro contra mancillado, alto contra bajo, claro contra oscuro, con la valoración positiva del primer término diádico y la valoración negativa del segundo.

El catolicismo institucional busca la separación de los católicos y sus prácticas, no tolera la mezcla de elementos heterogéneos de la religiosidad con su hipercodificación. Frente a la diversidad religiosa opta por la separación y la exclusión de lo diverso. Los esquemas verbales respectivos son lo puro contra lo mancillado. La pureza se da en la aceptación de las prácticas codificadas y su homogeneidad; el rechazo de las prácticas no codificadas y la heterogeneidad dan lugar a la impureza, visto como aquello que está fuera de lugar. Esto es el pecado, lo que viola la homogeneidad y lo que está fuera de la codificación. La claridad diurna no solo se expresa en la visión celeste del cielo y de Dios, sino en la diafanidad de la norma y el comportamiento regulado, con su respectiva angustia ante lo oscuro, lo refractario al control y la manipulación, dando lugar a una simbólica del mal. La siguiente cita aclara esta simbólica del mal:

La angustia y la muerte se atribuyen al devenir y al destino incontrolable, es así que el tiempo vivido se convierte en diversos tipos de engendros, representados mediante los símbolos terimorfos (animalidad, como el hombre lobo), nictormorfos (nocturnos, como brujas y fantasmas) y catamorfos (símbolos de la caída y el fracaso, como el destierro de Adán y Eva). Estos símbolos son monstruos terribles o situaciones peligrosas con connotaciones fuertemente femeninas. (Plascencia, 2017, p. 15)

Habría que añadir que estos símbolos se vinculan sobre todo con lo que no se puede ubicar en la lógica transparente, homogeneizante y racional del régimen diurno. Esto se manifiesta en la oposición entre lo claro y lo oscuro, tanto física como conceptualmente, pero también tiene que ver con la luminosidad diurna de un cielo que se encuentra en lo alto contra 
el infierno que se encuentra en lo bajo, o de lo oscuro propio de la noche y lo siniestro que constituye a los símbolos nictormorfos. El pecado es la caída tanto de Lucifer como de Adán y Eva, quienes por rebeldes y desobedientes se constituyen como arquetipos de los símbolos catamorfos. Además, lo alto no solo es el lugar del cielo y de Dios, es también el criterio que posiciona las cosas, las acciones y las personas; es el fundamento de la verticalización jerárquica establecida en manos de una institución monopólica: no se peca contra el cura o la Iglesia, se peca contra Dios con la heterodoxia.

El esquema, al relacionarse con el entorno, se sustantiviza y, al elegir una imagen concreta, enlazando lo imaginario con la intuición, da lugar al arquetipo. La dominante postural, el esquema distintivo de la disyunción excluyente, se combina con los arquetipos sustantivos del Dios celeste y omnipotente y superior contra el Diablo teriomorfo (emblema animalizado de lo diverso, lo caído y lo bajo); el cielo puro contra lo terrestre y la caída del pecado original; el bautismo que inscribe como fiel contra la mancha y la caída; el ángel armado (San Miguel) contra el monstruo teriomorfo (el dragón) o el disidente.

El reflejo da lugar al esquema. Este se sustantiviza en el arquetipo, y cuando se unen esquemas y arquetipos diversos se forman los símbolos. La iglesia es la constelación que unifica los valores-símbolos del Dios omnipotente, su jerarquía suprema y la pureza celeste; es la administradora del ascenso celeste por medio de los ritos y normas de inscripción, pertenencia, demostración, pureza y diafanidad de los objetos, las personas y las acciones. Todos estos son símbolos de los arquetipos y los esquemas del monopolio diurno y jerárquico de la Santa Madre Iglesia. Ahora que, cuando los fieles no están conscientes de toda la riqueza simbólica de, digamos, por ejemplo, un acto como el bautismo, que redime la caída, posibilita el ascenso, incluye en el esquema diferencial y excluye a todo lo que se mezcle, caiga, oscurezca y separe; entonces se produce el sistema, un símbolo debilitado al que no se le atribuyen los símbolos, arquetipos y esquemas respectivos.

Toma aquí sentido lo dicho por Grecia: "No creo en Dios enojado, creo en los santos". También tiene sentido la forma en que Eddy (creyente en la trascendencia) recrimina al catolicismo oficial: "Está muy bien que proporcionen valores, pero eso de que Dios te castiga, me cae de la fregada". El régimen diurno se concreta y hace que Dios aparezca como el héroe armado y polémico contra los "otros" (partidarios del aborto, del matrimonio homosexual, de los partidos de izquierda, etc.) a los que excluye y vence (desde la conveniencia y la realidad ideológica del grupo de referencia católico conservador).

El régimen nocturno, con sus dominantes nutritiva y cíclica, desde el que hablan Grecia, Eddy y sus coetáneos, en su dominante digestiva parte del descenso digestivo asociado con las materias de las profundidades como el agua y la tierra cavernosas. No es la espada, sino la copa que contiene, incluye y resguarda lo que suscita la asociación con el caldero y el cofre, con todo lo que contiene y protege. No es la técnica bélica, sino la alimenticia y la del brebaje que transforma nutritivamente, la que se asocia con los procesos simbólicos respectivos. Queda en el imaginario de los estudiantes el Padre Nuestro que proporciona el pan de cada día y que perdona las ofensas; de hecho, es la oración favorita de católicos, protestantes e incluso no creyentes entrevistados. Dios no excluye ni castiga, o bien, el "ser supremo" nos comprende a todos por igual y no amenaza ni reprime. Por otro lado, la concepción del ser supremo común entre los jóvenes es personal o impersonal, pero no necesariamente masculino. Valga la pena decirlo, el Dios diurno es masculino, represivo, 
excluyente y castigador de la disidencia. El ser supremo de los estudiantes no siempre es personal o masculino, ni castigador.

El esquema fundamental al que da lugar la dominante digestiva es la confusión contra la exclusión diurna. Confundir es mezclar y no excluir ni jerarquizar. Los esquemas verbales correspondientes son el descenso lento, el intimismo penetrante, la protección cálida. Descenso lento mas no caída, que iguala las cosas y las personas en una sana humildad. No es que no haya diferencias, es que a pesar de ellas no se producen ni jerarquías ni exclusión. Daniela, atea y anticlerical, tiene en su grupo de referencia de amigas a Fernanda, una escéptica con ribetes trascendentales, y a Lourdes, una católica practicante y miembro activo de la juventud católica. La tolerancia a la diversidad religiosa, sexual, social y política es lo habitual; mezclar y tolerar sin homologar ni excluir es lo normal y valioso. Un homosexual debe ser excluido dice la religión diurna, pero Ana Rosa, participante de un grupo juvenil católico, responde: "Si Dios no lo amara (a un homosexual), no lo hubiera hecho como lo hizo, es imposible, que Dios no te ame, por muy omnipotente que sea". Respecto del matrimonio homosexual: "Si es por amor, está bien hecho", dice Lourdes, otra influyente participante de los grupos de la juventud católica. La religión se antoja demasiado estrecha y represiva, fuera de época e insuficiente.

Los arquetipos relativos a la mezcla, el descenso suave y la protección son el ser supremo que incluye y recibe, la presencia terrestre de lo alto y el alimento y la protección del hogar y lo terrestre. Los símbolos-valor resultantes de los esquemas y arquetipos respectivos, que se mostraron en los estudiantes, dan cuenta de un ser supremo presente, inclusivo, nutricio y protector, que se sublima en trascendentalismos ecologistas (y paganos), como la tesis de Gaia y el biocentrismo. ${ }^{5} \mathrm{O}$ la visión "paternalista" del Padre Nuestro que perdona, protege, incluye y proporciona pan. Está también la visión de la muerte como retorno a la naturaleza y como descanso cálido y reconfortante en la eternidad. Pero ante todo hay una gran pregnancia de lo femenino que incluye, protege, alimenta y contiene uterina y cálidamente. Es la visión del ser supremo, como sucede en el catolicismo primitivo, pero la institucionalización del catolicismo la ha convertido en una visión patriarcal y autoritaria, semejante al héroe armado con la espada diurna contra la diversidad y la desobediencia.

La otra dominante nocturna, la copulativa, maduración del ritmo de la succión nutricia infantil, se desarrolla en la motricidad rítmica de los órganos humanos y en el coito reproductivo, con sus respectivos derivados sensoriales. El esquema dominante es la relación que permite la repetición, por lo que los arquetipos respectivos son el ciclo, la materialidad corporal, la segunda oportunidad y el progreso. Los símbolos respectivos son expresados por la libertad sexual y corporal, el cambio y el progreso. Al respecto, Jocelyne, feminista y creyente en un ser supremo, afirma lo siguiente: "Nadie tiene derecho a decirte cuál debe ser tu orientación sexual, el placer y la forma en la que decidas cómo lo vas a sentir en tu cuerpo; es personal, no como le pasó a mi tatarabuela". Ante la dominante postural, que separa y vence con las armas, y la digestiva, que protege y transforma para bien, la copulativa elige la

\footnotetext{
${ }^{5}$ La hipótesis de Gaia fue formulada por el químico inglés James Lovelock en 1969. Es una comprensión holística del planeta que forma la base del biocentrismo, doctrina que sitúa a la humanidad entre la totalidad de los seres vivos, con los que comparte tanto dignidad como relevancia funcional, y se ataca frontalmente al antropocentrismo, que considera al ser humano como destino de la evolución o centro de todas las cosas (o de la "Creación").
} 
repetición como promesa o segunda oportunidad. Frente a las dificultades que una atea (Daniela) y un católico heterodoxo (Cristóbal) tienen ante la sentencia católica de familiares: "Dios te castiga por hereje", la respuesta es diversa pero canónicamente cíclica: "Te pasan cosas, pero te repones, mañana saldrá el sol, aunque no lo quieran” (Daniela); “iQué va! Dios aprieta, pero no ahorca, ya le atinaré en la otra" (Cristóbal).

El régimen diurno no puede lidiar con el tiempo y el destino, la única certeza clara y distinta es la muerte; eso es observable, racional y transparente, propio del tiempo lineal diurno. La función vital de la imaginación reside en la eufemización -antífrasis de la temporalidad del destino humano y de la muerte-, la transformación creativa del mundo y de la muerte por asimilación a la vida. La imaginación niega la absurdidad y el sinsentido de la muerte y la existencia, y la torna aceptable con la dominante copulativa que niega la linealidad por medio del ciclo. El ecologismo documentado, por ejemplo, asimila la muerte a la vida en un ciclo de retorno y recíproca nutrición entre vivos y muertos, quienes se "nutren de" y "nutren a" alternadamente entre sí, en una reciprocidad solidaria y trascendente. Simultáneamente, subliman la sexualidad, la arrancan de toda ética represiva por medio del vitalismo y la igual importancia y bondad de todo proceso respectivo. Pero, sobre todo, se deconstruye la simbólica diurna represiva del mal. Los símbolos teriomórficos (representación animalizada del infractor) se cambian por el biocentrismo y la solidaridad con otras especies. Se relativizan los símbolos catamórficos (la caída como despeñadero moral y degradante) al dejar atrás la jerarquización institucional del culto y la moral represiva. Y a los nictomorfos (representación de lo diverso, lo incodificable y lo extraño como nocturno, oscuro y negativo) se les sustituye por la apertura y la tolerancia de la diversidad.

Un artículo sobre un movimiento carismático en Argentina (Olmos, 2017) muestra que la forma de representar la religión como institución, a Dios y a los ministros, son síntomas de la emergencia de "nuevas formas de habitar el catolicismo". Basándonos en este artículo, podemos pensar que la forma de representación de Dios y la Iglesia católica que hemos documentado, puede dar lugar a una nueva forma de catolicismo, más allá de lo meramente contestatario y quizá sentar una esperanza para el futuro.

El régimen nocturno, en su dominante copulativa, aporta la ideación y el valorsímbolo más importante de nuestro amplio y complejo grupo estudiado: la esperanza. La confianza en el futuro, el porvenir y la segunda oportunidad de una mezcla de seres tan valiosos como diversos y dignos de respeto; lugar y futuro en el que no merecen ser objeto de la represión, la exclusión y la discriminación por parte de quienes se creen depositarios institucionales de lo sacro, lo verdadero, lo moral, lo políticamente correcto y lo bello. Los estudiantes hablaron y prestaron sus voces a ateos, católicos domingueros o "cucarachas de Iglesia”, a los machos, lesbianas y bisexuales; a políticos prianistas, morenistas y demás. Dejaron constancia de que para ellos cada quien puede ser lo que sea, pero sobre todo de que tiene derecho y debe ser apoyado para convertirse en lo que quiere y -precisamente por esodebe ser. Por eso será apoyado desde la amplia comunidad de pensamiento, acción y percepción de sus iguales. 


\section{Conclusiones}

El grupo de referencia etario de la religiosidad, de los estudiantes de licenciatura de la UAA, constituye un colectivo de jóvenes que comparten un conjunto de valores -símbolos construidos en torno a la apertura, la solidaridad, la mezcla y la esperanza, propios de un régimen simbólico nocturno- desde los cuales reflexionan, perciben, actúan y construyen un modo de elaboración de lo sacro correspondiente: la religiosidad.

Desde la religiosidad, planteamos la hipótesis: los estudiantes construyen también un modelo vital que se opone a todas las instituciones propias del régimen simbólico diurno, las cuales son vistas como grupos de referencias negativos a los que no se desea pertenecer en diversos grados de rechazo. Hay católicos, pero solo en menor grado de incorporación y asentimiento: son no practicantes y prefieren la experiencia directa sin el ritual de la misa. Hay ateos, quienes, sin embargo, piensan en una trascendencia ecológica o en un orden inteligente y supremo que incluye valores de apertura, respeto y solidaridad.

Los símbolos de la degradación y la descalificación del régimen diurno represivo de la Iglesia católica -catamorfos, nictomorfos y teriomorfos-, derivados de la insuficiencia de la razón frente a la irreversibilidad del tiempo y la muerte y de los límites del control represivo, son combatidos por el régimen nocturno. Los símbolos catamorfos representación oprobiosa de la caída por la insurrección- son reflexivamente inhabilitados, tanto por la inexistencia de la mancha, en razón de atentar contra la jerarquía y la norma represiva respectiva, como por la imagen de la caída suave y homologante que incluye el blando descenso a la eternidad en la morada terrestre al morir.

Los símbolos nictomorfos -figuración de lo siniestro por inclasificable y oscuro-son sustituidos por la serenidad nocturna y la tolerancia frente a lo que no es claro ni diáfano, por lo que no es definido, pero sí valioso.

Contra los símbolos teriomorfos, la bestialización de lo incontrolable y sin domesticación, se apuesta por no animalizar lo diverso ni degradar a otras especies. La teriomorfización diabólica es miedo ante lo diverso y lo desconocido. El mal es muy humano como para atribuirlo a otras especies; esta teriomorfización se ve injusta, equivocada y paranoica en la representación de los estudiados. Finalmente, el tiempo es maduración y esperanza de lo diverso. Todo esto, según el régimen nocturno simbólico de la religiosidad etaria aplicado a las concepciones manifiestas en los testimonios recogidos de los estudiantes de licenciatura de la UAA, en el marco de una investigación cuyo objetivo general era mostrar cómo se perfiló el sistema de creencias religiosas de los estudiantes en un sistema simbólico.

\section{Referencias bibliográficas}

Abreu, C. (2012). La teoría de los grupos de referencia. Papeles de Filosofía, 31(2), 287-309. Abric, J. C. (ed.) (1994). Pratiques sociales et représentations. París: Presses Universitaires de France.

Arnett, J. (2008). Adolescencia y adultez emergente. Un enfoque cultural. México: Pearson. Bourdieu, P. (1997). Capital cultural, escuela y espacio social. México: Siglo XXI Editores. Bourdieu, P. (2002). Condición de clase y posición de clase. Revista Colombiana de Sociología, 7(1), 119-141.

Bourdieu, P. (2006). Génesis y estructura del campo religioso. Relaciones, 27(108), 29-83. 
Bourdieu, P. y Passeron, J. C. (2003). Los herederos. Los estudiantes y la cultura. Buenos Aires: Siglo XXI Editores.

Chami, D. L., Scudieri, M. e Imhoff, D. (2018). Abordaje psicosocial de la identidad judía en jóvenes de una escuela de la colectividad masortí de Córdoba, Argentina. Revista Cultura y Religión, 12(1), 4-27.

Durand, G. (2004). Las estructuras antropológicas del imaginario. Introducción a la arquetipología general. México: FCE.

Durkheim, E. (1968). Las formas elementales de la vida religiosa. Buenos Aires: Schapiro.

Ellis, C., Adams, T. E. y Bochner, A. P. (2010). Autoethnography: An Overview [40 paragraphs]. Forum Qualitative Sozialforschung/Forum: Qualitative Social Research, 12(1), Art. 10. Recuperado de http://nbn-resolving.de/urn:nbn:de:0114fqs 1101108 [Consultado el 12 de julio de 2019].

Ferrarotti, F. (1993). Una fe sin dogmas. Barcelona: Ediciones Península.

Giménez, G. (1999). La importancia estratégica de los estudios culturales en el campo de las ciencias sociales. En C. R. Reguillo y N. R. Fuentes, Pensar las ciencias sociales hoy (pp.72-96). México. ITESO.

Freud, S. (2005). La interpretación de los sueños. México: Origen-Planeta.

Geertz, C. (1987). La religión como sistema cultural. La interpretación de las culturas (pp. 87-117). México: Editorial Gedisa.

González, J. (2 de octubre de 2018). Aguascalientes: Obispo acusa de 'aberraciones' a defensores del aborto legal. Expansión política. [En línea]. Recuperado de https://politica.expansion.mx/mexico/2018/10/02/obispo-de-aguascalientes-llamadesnivelados-a-promoto [Consultado el 18 de junio de 2019].

INEGI (2010). Censo de Población y Vivienda 2010. Recuperado de https://www.inegi.org.mx/programas/ccpv/2010/ [Consultado el 3 de octubre de 2016].

Jodelet, F. (1989). Les preprésentations sociales. París: Presses Universitaires de France.

Merton, R. K. (2002a). Aportaciones a la teoría de la conducta del grupo de referencia. Teoría y estructura sociales (pp. 305-361). México: Fondo de Cultura Económica.

Merton, R. K. (2002b). Continuidades en la teoría de los grupos de referencia y la estructura social. Teoría y estructura sociales (pp. 362-470). México: Fondo de Cultura Económica.

Miles, M. y Huberman, H. (1986). Qualitative Data Analysis. A Sourcebook of New Methods. Beverly Hills, Cal.: SAGE Publications.

Olmos, A. L. (2017). Otro catolicismo posible: institución, dios y agentes católicos en las experiencias biográficas de las fieles. Revista Cultura y Religión, 11(1), 4-22.

Patiño, M. E. (coord.) (2013). Creencias y prácticas religiosas en Aguascalientes. Ensayos interpretativos. Aguascalientes, Ags.: Universidad Autónoma de Aguascalientes (UAA).

Patiño, M. E. (2014). Panorama sobre los estudios sobre la diversidad católica en México: apuntes para su reflexión. Revista Cultura y Religión, 8(1), 132-150.Plascencia, F. (2017). La función simbólica en la interpretación del mundo. Una introducción para zombis. Aguascalientes, Ags.: UAA. 
Ros, J. (2018). Claves para una sociología de la experiencia religiosa en el ámbito católico. Revista Cultura y Religión, 12(1), 54-74.

Sánchez, O. y Plascencia, F. (2017). La religión como conocimiento cultural: el caso de los estudiantes de licenciatura en la Universidad Autónoma de Aguascalientes en México. Revista Cultura y Religión, 11(2), 29-50.

Sánchez, O., Plascencia, F. y Alemán, R. (2018). Marx, expectativas familiares de estudiantes de clases bajas y la acción transformadora en universitarios de ciencias sociales. Congreso: La obra perdurable de Marx a 200 años de su muerte, 8 de junio, Universidad Autónoma de Zacatecas, Zacatecas, México.

Sánchez, O., Plascencia, F. y Tapia, E. (2016-2017). Capital cultural, jóvenes universitarios y aprovechamiento escolar. Proyecto de investigación, UAA. (Inédito)

Sánchez, O., Plascencia, F. y Tapia, E. (2017). Capital cultural, jóvenes universitarios y aprovechamiento escolar. Informe final de investigación. Aguascalientes, Ags.: UAA.

Sánchez, O., Plascencia, F. y Tapia, E. (2018). Incidencia de los grupos de referencia en la trayectoria académica de estudiantes del Centro de Ciencias Sociales y Humanidades de la UAA. Primer informe anual de investigación, UAA.

Sánchez, O., Plascencia, F. y Tapia, E. (2018-2020). Incidencia de los grupos de referencia en la trayectoria académica de estudiantes del Centro de Ciencias Sociales y Humanidades de la UAA. Proyecto de investigación: PIECU18-6, financiamiento interno UAA, UAA.

Spradley, J. P. (1979). The Ethnographic Interview. Nueva York: Holt, Rinehart \& Winston. Thompson, J. (1993). Ideología y cultura moderna. México: UAM-Xochimilco.

Zalpa, G. (2006). Estudio comparativo de las concepciones sobre la familia en algunas iglesias de Aguascalientes. Informe final de investigación, UAA.

Zalpa, G. (2002). El campo religioso en la diócesis de Aguascalientes. Aguascalientes, Ags.: UAA.

Cómo citar este artículo

Sánchez, O.; Plascencia, F. y Berna, L. (2020). Grupo de referencia etario, régimen simbólico y religiosidad. La religiosidad como sistema abierto de creencias en estudiantes universitarios. Revista Cultura \& Religión, 14(2), 121-140. 